Der Tübinger Historiker Anselm Doering-Manteuffel hat in den Vierteljahrsheften für Zeitgeschichte vor kurzem sein Konzept der Zeitbögen vorgestellt. Er bietet damit ein politische Zäsuren übergreifendes, an handlungssteuernden Ideen orientiertes Deutungsmuster an, um die deutsche Geschichte im 20. Jahrhundert zu verorten. Peter Hoeres nimmt das Konzept der Zeitbögen kritisch unter die Lupe, hinterfragt seine normativen Implikationen, wägt analytisches Potenzial und inhaltlich-methodische Blindstellen gegeneinander ab und zeigt Perspektiven für alternative Interpretationen auf.

Peter Hoeres

\title{
Gefangen in der analytisch-normativen Westernisierung der Zeitgeschichte
}

Eine Kritik am Konzept der Zeitbögen

Wie ist die wechselvolle deutsche Geschichte des 20. Jahrhunderts historiografisch konzeptualisierbar? Kann man sie wie Heinrich August Winkler und Ulrich Herbert, mit stabilem normativen Werkzeug ausgestattet, in zwei Hälften mit einer mehr (Winkler) oder weniger (Herbert) scharfen Zäsur 1945 teilen ? Der Vorschlag, den jüngst Anselm Doering-Manteuffel an dieser Stelle gemacht hat ${ }^{2}$, ist anspruchsvoller. Anders als Winkler und Herbert, die in ihren Gesamtdarstellungen in unterschiedlichem Maße auf die politischen Zäsuren fixiert bleiben, orientiert sich Doering-Manteuffel an Zeitbögen. Unter dem ursprünglich astronomischen Begriff versteht der Autor, der über eine ausgeprägte Fähigkeit zur Begriffsprägung verfügt ${ }^{3}$, eine Zeitspanne von mehreren, sich überlappenden Dekaden, die „durch markant unterschiedliche Vorstellungen von der Ordnung in Gesellschaft und Staat gekennzeichnet sind“ (S. 324). Das Konzept gilt es im Folgenden kritisch zu diskutieren und mit Alternativvorschlägen zu konfrontieren. Damit sollen einige Probleme einer analytisch-normativen Westernisierung

\footnotetext{
${ }^{1}$ Vgl. Heinrich August Winkler, Der lange Weg nach Westen, 2 Bde., München 2000; ders., Geschichte des Westens, Bd. 3: Vom Kalten Krieg zum Mauerfall, München 2014; Ulrich Herbert, Geschichte Deutschlands im 20. Jahrhundert, München 2014. Herbert geht zwar von einer historischen Einheit der „Hochmoderne“ zwischen 1890 und 1990 aus, hält aber an einer scharfen Zäsur der „Stunde Null“ (S. 549, dort ohne Anführungszeichen) fest. Zu Herberts Buch vgl. meine demnächst erscheinende ausführliche Kritik im Jahrbuch Extremismus \& Demokratie 27 (2015).

${ }^{2}$ Vgl. Anselm Doering-Manteuffel, Die deutsche Geschichte in den Zeitbögen des 20. Jahrhunderts, in: VfZ 62 (2014), S. 321-348. Die mit Seitenzahlen im Haupttext ausgewiesenen Zitate im Folgenden stammen aus diesem Aufsatz.

${ }^{3}$ Vgl. Anselm Doering-Manteuffel, Wie westlich sind die Deutschen? Amerikanisierung und Westernisierung im 20. Jahrhundert, Göttingen 1999; ders./Lutz Raphael, Nach dem Boom. Perspektiven auf die Zeitgeschichte seit 1970, Göttingen 2008.
} 
der Zeitgeschichtsschreibung erörtert werden, von der auch die Synthesen von Winkler und Herbert bestimmt sind.

I.

Die Vorzüge der Zeitbögen sind einleuchtend ${ }^{4}$ : Sie unterlaufen erstens die politischen Zäsuren, die auf viele Zeitgenossen zunächst gar keine Auswirkungen hatten. Für die Westdeutschen änderte sich mit dem 9. November 1989 oder dem 3. Oktober 1990, wenn sie nicht unmittelbar an der innerdeutschen Grenze wohnten, beispielsweise zunächst wenig. Und dass selbst der 8. Mai 1945 keine Stunde Null war, ist evident. Mit Blick auf sozialhistorische Entwicklungen in der NS-Zeit und deren Folgewirkungen hatte Martin Broszat bereits 1985 eine „periodenübergreifende Betrachtung“" gefordert, ein Postulat, das bisher noch nicht eingelöst ist ${ }^{5}$. Die politischen Zäsuren werden mit dem Konzept der Zeitbögen nicht leichtfertig eskamotiert, aber der Blick darauf verändert sich. Zweitens können die Zeitbögen längerfristige Entwicklungen markieren und identifizieren, ohne dass die Dekadeneinteilung durch überlange 1960er Jahre oder sehr kurze 1970 er und 1980er Jahre überstrapaziert werden muss. Drittens tragen die Zeitbögen tatsächlich zu einer „Gesellschaftsgeschichte handlungssteuernder Ideen“ (S. 324) bei, ein Ansatz, um den sich Doering-Manteuffel im Rahmen der „neuen Ideengeschichte" außerordentlich verdient gemacht hat.

Worin bestehen nun die Zeitbögen, welche die „euroatlantischen Industriegesellschaften“ (S.324) des vergangenen Jahrhunderts bestimmt haben sollen? Der erste umfasst die Hochindustrialisierung von 1890 bis zum Zweiten Weltkrieg. Dieser Zeitbogen ist nur wenig profiliert, denn die Zeitbögen zielen auf einen weiten „europäisch-atlantischen Horizont“ (S. 322), vor dem nationale Besonderheiten erkennbar werden sollen. Die Krise des Liberalismus und des Bürgertums, die Doering-Manteuffel als Signatur seines ersten Zeitbogens diagnostiziert, soll ein „europäisches Phänomen“ (S. 326) darstellen; nicht auch - diese Frage drängt sich auf - ein amerikanisches? Die „Kulturrevolution des Antiliberalismus“ (S.329) ist dagegen offenbar ein primär deutsches Phänomen. Durch den affirmativen Verweis auf Thorstein Veblen wird bereits deutlich, dass Doering-Manteuffel implizit an der Sonderwegsthese festhält, auch mit Blick auf einen spezifischen eliminatorischen Antisemitismus, Daniel Goldhagen wird freilich nicht genannt. „Der Gegensatz zwischen liberalen und antiliberalen Vorstellungen über die Ordnung von Gesellschaft und Staat“ sei nun, so der Autor weiter, zwischen 1914 und 1918 von der „Kriegspropaganda aller Seiten ins Grundsätzliche vertieft" (S. 328) worden. Das heißt aber doch wohl, dass dieser Gegensatz, weniger grundsätzlich als es in der Propaganda hieß, durchaus bestand. Angesichts der

\footnotetext{
${ }^{4}$ Doering-Manteuffels eigene Beschreibung des analytischen Potentials der Zeitbögen, die „Sichtbarmachung der Mehrdimensionalität des historischen Geschehens“ (S. 325), scheint mir nicht besonders aussagekräftig zu sein.

${ }^{5}$ Martin Broszat, Plädoyer für eine Historisierung des Nationalsozialismus, in: Merkur 39 (1985), S. 373-385, Zitat S. 384.
} 
neuen Forschungen zum Kaiserreich und zum Ersten Weltkrieg ist dieser Gegensatz als Charakterisierung einer Differenz zwischen dem Deutschen Reich und anderen europäischen Staaten jedoch nicht mehr zu halten, weder für die Vorkriegs- noch für die Kriegszeit, weder für die politische Kultur noch für die grundsätzlichen Ordnungsmodelle ${ }^{6}$. Mit Blick auf die (visuelle) Kriegspropaganda zeigt sich zudem, dass die Bestialisierung und Dehumanisierung des Feindes eine vor allem alliierte Angelegenheit (besonders Frankreichs und der USA) gewesen ist und im Deutschen Reich nach einem von der Obersten Heeresleitung bestellten Rechtsgutachten sogar untersagt war. Auch die Handhabung von Zensur und Repression gegen Kriegsgegner und „Dissenters“ war bei den Staaten der Entente mindestens ebenso „antiliberal“ wie bei den Mittelmächten ${ }^{7}$. Der Gegensatz von liberalen und antiliberalen Vorstellungen firmiert bei Doering-Manteuffel dann aber zugleich als innerdeutscher Gegensatz, der die Weimarer Republik bestimmte. Die dezidiert antiliberalen „konservativen Revolutionen“ anderer Länder lässt Doering-Manteuffel trotz zahlreicher Studien der letzten Jahre zu diesem Thema außer Acht ${ }^{8}$.

Deutlicher konzeptualisiert wird der von sozialem Konsens, Gleichheit und Gemeinschaft definierte zweite Zeitbogen von 1930 bis 1973/75. Dies ist der Zeitbogen des Keynesianismus, mit einer starken Orientierung an den USA, an Fordismus und Taylorismus, am New Deal. Soziale Integration, Gleichheit und Homogenität waren die Leitvorstellungen. Hier wird die Dichotomie von liberal/ antiliberal einmal aufgebrochen, wenn Doering-Manteuffel die Orientierungsfunktion der USA auch für das Dritte Reich profiliert. Dass der Nationalsozialismus aber primär als Antiliberalismus und Antirationalismus verstanden wird der Antibolschewismus wird ebenso marginalisiert wie die Dialektik des Rationalismus ${ }^{9}$-, läuft auf eine ungute Verbindung von Georg Lukács ${ }^{10}$ und WhigHistory hinaus. Das Ende des lang anhaltenden Nachkriegsbooms in den 1970er Jahren schließt den zweiten Zeitbogen ab.

\footnotetext{
${ }^{6}$ Vgl. synthetisierend Frank Lothar Kroll, Geburt der Moderne. Politik, Gesellschaft und Kultur vor dem Ersten Weltkrieg, Berlin 2013. Zu den strukturellen ideengeschichtlichen Parallelen des deutschen und britischen Diskurses der Kriegszeit siehe Peter Hoeres, Krieg der Philosophen. Die deutsche und die britische Philosophie im Ersten Weltkrieg, Paderborn 2004.

${ }^{7}$ Vgl. Dieter Vorsteher, Bilder für den Sieg. Das Plakat im Ersten Weltkrieg, in: Rainer Rother (Hrsg.), Die letzten Tage der Menschheit. Bilder des Ersten Weltkrieges, Berlin 1994, S. 149-162; Adam Hochschild, To end all Wars. A Story of Loyalty and Rebellion, 1914-1918, Boston 2011; Charles Ridel, Propaganda at Home (France), in: Ute Daniel u.a. (Hrsg.), 1914-1918-online. International Encyclopedia of the First World War, Berlin 2014, DOI: 10.15463/ie1418.10359; Lon Strauss, Social Conflict and Control, Protest and Repression (USA), in: Ebenda, DOI: 10.15463/ie1418.10281.

${ }^{8}$ Vgl. Bernhard Dietz, Neo-Tories. Britische Konservative im Aufstand gegen Demokratie und politische Moderne 1929-1939, München 2012; Hans-Wilhelm Eckert, Konservative Revolution in Frankreich? Die Nonkonformisten der Jeune Droite und des Ordre Nouveau in der Krise der 30er Jahre, München 2000.

${ }^{9}$ Zur Wiederlektüre vorgeschlagen sei Max Horkheimer/Theodor W. Adorno, Dialektik der Aufklärung. Philosophische Fragmente, Frankfurt a. M. 1988 [zuerst 1947].

${ }^{10}$ Vgl. Georg Lukács, Die Zerstörung der Vernunft, Berlin 1954.
} 
Der dritte Zeitbogen beginnt folgerichtig in den frühen 1970er Jahren und ist im Prinzip noch nicht beendet. Doering-Manteuffel sieht ihn bestimmt von dem Bedeutungsverlust der Nationalstaaten in der Globalisierung, von der Individualisierung, vor allem aber von der Neujustierung des Freiheitsbegriffs als „Freiheit des Marktes“ (S. 343) vom Staat. Der „Neoliberalismus als konservatives Projekt“ (S.341) - eine angesichts der ordoliberalen Ursprünge ganz verfehlte Bezeichnung für „Marktradikalismus“ - habe sogar zu einer „Abkehr von der sozialdemokratischen Tradition der Staatsorientierung“ (S.348) geführt, wie Doering-Manteuffel mit dem Hinweis auf das „Schröder-Blair-Papier“ von 1999 glaubt belegen zu können.

Das ist nun alles andere als überzeugend. Kommen wir zunächst zum immerhin an einer Stelle kurz problematisierten Begriff „Neoliberalismus“ (S.346). Doering-Manteuffel ignoriert die laufende Debatte über die Verwendung zeitgenössischer Selbstbeschreibungen in der Geschichtswissenschaft ${ }^{11}$, die sozialwissenschaftliche Gegenwartsanalysen und deren Wert für die Geschichtswissenschaft problematisiert. Beim Schlagwort „Neoliberalismus“ kommt verschärfend hinzu, dass es sich um einen polemisch konnotierten Begriff der Publizistik handelt. Gerade eine gegenwartsnahe Zeitgeschichtsschreibung muss hier besonders auf der Hut sein und publizistisch verwendete Begriffe befragen. Die Ordoliberalen Alexander Rüstow und Wilhelm Röpke haben diesen Begriff seit den 1930er Jahren bisweilen verwendet, zum Leidwesen anderer Ordoliberaler wie Erich Welter, der vor diesem Schlagwort warnte ${ }^{12}$. Deregulierung, „Marktradikalismus“ und die Herrschaft der Finanzmärkte waren damit nicht gemeint. Aber auch inhaltlich taugt der Begriff kaum zur Beschreibung des dritten Zeitbogens. Gerade die Konjunktur des polemischen, heutzutage rein pejorativ gebrauchten Begriffs ist eher ein Anzeichen für die verbreitete Gegnerschaft. Auch die Befunde sind bei näherer Betrachtung keineswegs eindeutig.

Weder hat sich die SPD das "Schröder-Blair-Papier“ dauerhaft zu eigen gemacht, noch hat die Agenda-Politik den Anteil der Sozialausgaben am Staatshaushalt und am Bruttoinlandsprodukt nennenswert gesenkt. Man braucht nicht erst auf die Maßnahmen der Großen Koalition III, die Rente mit 67 und die Mütterrente, hinzuweisen und man braucht nicht eigens auf die Steuererhöhungen unter François Hollande in Frankreich und die Fiskalpolitik der Europäischen

${ }^{11}$ Vgl. aus der Debatte: Rüdiger Graf/Kim Priemel, Zeitgeschichte in der Welt der Sozialwissenschaften, in: VfZ 59 (2011), S. 479-508; Benjamin Ziemann, Sozialgeschichte und empirische Sozialforschung, in: Pascal Maeder/Barbara Lüthi/Thomas Mergel (Hrsg.), Wozu noch Sozialgeschichte? Eine Disziplin im Umbruch, Göttingen 2012, S. 131-149; Bernhard Dietz/ Christopher Neumaier, Vom Nutzen der Sozialwissenschaften für die Zeitgeschichte. Werte und Wertewandel als Gegenstand historischer Forschung, in: VfZ 60 (2012), S. 293-304; Andreas Rödder, Wertewandel in historischer Perspektive. Ein Forschungskonzept, in: Bernhard Dietz/Christopher Neumaier/Andreas Rödder (Hrsg.), Gab es den Wertewandel? Neue Forschungen zum gesellschaftlich-kulturellen Wandel seit den 1960er Jahren, München 2014, S. 17-39.

${ }^{12}$ Vgl. Peter Hoeres, „Frankfurter Allgemeine Langeweile?“, in: Frankfurter Allgemeine Zeitung vom 21. 10. 2014, S. 22f. Eine Biografie Erich Welters wird derzeit von meiner Doktorandin Christina Schäfer erarbeitet. 
Zentralbank zu schauen, um festzustellen, dass es gerade keine neoliberale Agenda ist, die den Kurs in der Bundesrepublik und der Europäischen Union bestimmt. Die Große Koalition II betrieb mit der „Abwrackprämie“ 2009 eine antizyklische Konjunkturpolitik wie aus einem Lehrbuch des Keynesianismus. Die gigantischen Transfers im Zuge der Euro-Rettung, die Ausweitung der Armutsmigration, aber auch die immer durchgreifenderen Versuche, die Gesellschaft nach politischen Vorstellungen und Vorgaben $\mathrm{zu}$ formen, konterkarieren eine vermeintliche neoliberale Signatur, die nur für wenige Politikfelder zu beobachten ist: bei der Privatisierung der Staatsunternehmen (VEBA, Lufthansa, Bundespost, Deutsche Bahn), der vorsichtigen Flexibilisierung des Arbeitsmarkts durch das Beschäftigungsförderungsgesetz (1985) und der Steuerreform von 1988 in der Ära Kohl ${ }^{13}$ sowie bei der rot-grünen Deregulierung der Finanzmärkte zwischen 2002 und $2004^{14}$, die nun zum Teil rückgängig gemacht wird. Dies waren allesamt Maßnahmen, die durch gegenläufige Ausweitungen des Sozialstaates flankiert oder überdeckt wurden. Auch die Agenda 2010 selbst mit den HartzGesetzen kann schwerlich als neoliberal im Sinne des heutigen Sprachgebrauchs interpretiert werden; sie hat sogar zu einer Ausweitung der Arbeitsmarktverwaltung und der Transferleistungen geführt ${ }^{15}$.

Auch für die neoliberalen „Modellstaaten“ bietet sich ein disparates Bild. Selbst in den USA unter Ronald Reagan wurde die Staatsquote nicht gesenkt, und der Umfang der Bundesbeschäftigten stieg entgegen der Rhetorik („government is not the solution to our problem, government is the problem") sogar noch $\mathrm{an}^{16}$. Unter Barack Obama erlebten die USA mit dem American Recovery and Reinvestment Act von 2009 eine Renaissance des Keynesianismus ${ }^{17}$. Und die Nach-Thatcher-Ära mit New Labour in Großbritannien war auch von Versuchen geprägt, mit der Einführung eines landesweiten Mindestlohns, eines Gesetzes zur betrieblichen Anerkennung von Gewerkschaften und weiteren Schutzmaßnahmen von Arbeitnehmern- und Gewerkschaftsrechten sozial- und gesellschaftspolitisch zu

\footnotetext{
13 Vgl. Andreas Wirsching, Abschied vom Provisorium 1982-1990, München 2006, S. 255-264 u. S. 277-288. Die Postreform der 1980er Jahre wird von Gabriele Metzler aber plausibel gerade nicht als Ausdruck neoliberaler Ideologie verstanden, vielmehr war sie nicht zuletzt von sozialstaatlichen Prämissen geprägt; vgl. Gabriele Metzler, Die Liberalisierung der Telekomunikation in der Bundesrepublik, in: Meik Woyke (Hrsg.), Wandel des Politischen. Die Bundesrepublik Deutschland während der 1980er Jahre, Bonn 2013, S. 167-194.

14 Die Wertung Edgar Wolfrums, Rot-Grün an der Macht. Deutschland 1998-2005, München 2013, S.523f., Rot-Grün habe den globalen Deregulierungstrend eher bremsend begleitet, folgt sehr stark der rückblickenden Einschätzung eines damaligen Akteurs, nämlich Hans Eichels, Dichtung und Wahrheit, in: Süddeutsche Zeitung vom 31.8.2012.

${ }^{15}$ Vgl. Simon Hegelich/David Knollmann/Johanna Kuhlmann, Agenda 2010. Strategien - Entscheidungen - Konsequenzen, Wiesbaden 2001, S. 131. Allein die Anzahl ganzer Stellen der Bundesagentur für Arbeit (Vollzeitäquivalente) stieg von 83.000 (2005) auf 106.000 (2010) an, danach sank sie wieder, ist aber noch deutlich über dem Niveau von 2005. Freundliche Auskunft der Bundesagentur vom 4. 12. 2014.

${ }^{16}$ Michael Schaller, Ronald Reagan, Oxford 2011, S. 34 u. S. 46. Das Zitat stammt aus der Inaugural Address vom 20.1.1981.

17 Vgl. Christiane Lemke, Richtungswechsel. Reformpolitik der Obama-Administration, Wiesbaden 2011, S. 55-71.
} 
intervenieren. Damit hat New Labour im neoliberalen Zeitbogen „auch einen Beitrag zur Erfüllung jenes historischen sozialdemokratischen Ziels geleistet, den Beschäftigten Schutzmöglichkeiten vor der Macht der privaten Unternehmer zu bieten"18.

Wenn man eine „Gesellschaftsgeschichte handlungssteuernde[r] Idee[n]“ anstrebt, müsste man für Deutschland die Gründung und Stabilisierung der Linkspartei als Kontrast zum „Schröder-Blair-Papier“ wenigstens erwähnen. Auch in den Wirtschaftswissenschaften finden sich zahlreiche Gegenströmungen zum Neoliberalismus beziehungsweise zur Neoklassik. Ob angesichts der Diskurshegemonie der Verfechter der sozialen Gerechtigkeit ein „Wandel in der Politik vom Primat des sozialen Konsenses zum Primat des Subjektivismus“ (S. 345) konstatiert werden kann, ist also mit einem großen Fragezeichen zu versehen.

Gerade dieser letzte Zeitbogen führt zu einer Reduktion an gewünschter Mehrdimensionalität, zu einer falschen Eindeutigkeit in der Beobachtung langfristiger Tendenzen. Dahinter liegen größere Probleme der Konzeptualisierung der Zeitbögen, die in einer doppelten, nämlich analytischen und normativen Privilegierung der „Westernisierung“ zu liegen scheinen. Die deutsche Geschichte wird als Teleologie hin zu einem „Platz im atlantischen Ordnungssystem“ verstanden (S. 348) und auf eine „Teilhabe am oder der Gegnerschaft zum westlich-liberalen Ordnungsmodell“ (S.324) befragt. Könnte es nicht überraschende Ergebnisse zeitigen, einmal nach deutscher Teilhabe an östlichen Ordnungsmodellen zu fragen? Oder nach westlicher Teilhabe an deutschen wissenschaftlichen, kulturellen, wirtschaftlichen und sozialpolitischen Ordnungsmodellen?

Der Preis der „Westernisierung“ der Leitfragen und Kategorien ist in analytischer Hinsicht ein Schematismus der Zeitbögen - eine Homogenisierung und normativ eine Idealisierung des „Westens“. Dass in der Weimarer Republik relativ gesehen de facto mehr Bürger wählen konnten als in den USA vor dem Voting Rights Act 1965, dass die Klassengesellschaft sich in Deutschland nie so scharf ausprägen konnte wie in Großbritannien, dass die soziale Schere in Deutschland nicht so weit wie in den USA auseinander ging - all das wird ausgeblendet. Den Preis der Verkürzung zeigen dann apodiktisch vorgetragene Fehlurteile im Einzelnen, mit denen man wieder beim eigentlich doch recht konsensual verabschiedeten Sonderweg anlangt. Die Entwürfe von Winkler, Herbert und Doering-Manteuffel sind in diesem Punkt eben gerade nicht alternativ, sie sind auch nicht transnational oder global perspektiviert.

\section{II.}

Einige Fehlschlüsse und Fehlurteile, zu der die analytisch-normative Doppelorientierung an der Westernisierung führt, sollen im Folgenden exemplarisch

${ }^{18}$ Steve Ludlam, New Labour, Arbeitsmarktpolitik und Arbeitsbeziehungen, in: Hans Kastendiek/Roland Sturm (Hrsg.), Länderbericht Großbritannien. Geschichte - Politik - Wirtschaft - Gesellschaft - Kultur, Opladen 2007, S.456-477, Zitat S.471. Siehe dort auch die Übersicht über die umfangreiche beschäftigungspolitische Gesetzgebung 1998-2004, S. 470. 
angedeutet werden. Insgesamt fällt auf, dass selbst gewagte Aussagesätze offenbar nicht als begründungspflichtig angesehen werden. Gleich zu Beginn heißt es bei Doering-Manteuffel, dass das Zeitalter der Nationalstaaten vorbei sei (S. 321). Das hat man nun aus der Politologie und Soziologie schon so oft gehört, dass man sich fragt, warum sich die Welt an diese Vorgabe einfach nicht halten will. Die zentralen Akteure gerade in Krisenzeiten sind dann doch immer wieder die Nationalstaaten, die sich freilich verändern, aber im Zusammenspiel mit anderen Akteuren - einstweilen - die entscheidende Instanz zur Herstellung kollektiv verbindlicher Entscheidungen bleiben ${ }^{19}$. Analytisch weiterführender als solche apodiktischen Vorgaben wäre es gewesen, die Prozesse der Entnationalisierung (Zweistaatlichkeit, Verblassen der nationalen Orientierung), der Globalisierung und Renationalisierung (im Zuge der Neuen Ostpolitik ${ }^{20}$, der konservativliberalen Wende 1982 und der Wiedervereinigung) der deutschen Geschichte im 20. Jahrhundert zu verfolgen und zu ergründen.

Dass der Versailler Vertrag „dem Deutschen Reich die Möglichkeit [eröffnete], sich wirtschaftlich, politisch und ideologisch neu zu festigen“ (S. 323), ist ein Euphemismus, welcher den Vätern des Vertrags vollkommen fremd war ${ }^{21}$. Differenziert oder gar begründet ist das Urteil jedenfalls nicht ${ }^{22}$. Dass der Nationalsozialismus keine Verbindung mehr zum westlichen Rechtsdenken besessen habe, ist ebenso falsch. Die Versuche, eine deutsche Monroedoktrin zu entwerfen, zeigen anderes, wenn diese auch von Fehldeutungen bestimmt war ${ }^{23}$. Unscharf ist die an Hermann Rauschning erinnernde Formel von der „nihilistischen Utopie des Nationalsozialismus" (S.332) ${ }^{24}$. Inwiefern kann eine Utopie überhaupt nihilistisch sein? Die Formel verweist allenfalls auf Deutungsmuster der 1950er Jahre, welche im Nationalsozialismus eine nihilistische Abkehr von Gott und der Tradition ausmachten. Das ist hier aber offenkundig nicht gemeint; was aber dann? Gravierender und bezeichnender ist die wiederum in Form eines apodiktischen Aussagesatzes formulierte These, der „Zweite Weltkrieg, ja überhaupt die Zeit des

${ }^{19}$ Vgl. Werner Abelshauser, Geschichte wiederholt sich nicht. Oder doch? Szenarien der Finanzmarktkrise, in: Zeitschrift für Staats- und Europawissenschaften 4 (2008), S. 565-576.

${ }^{20}$ Diese wurde jedenfalls in weiten Teilen des Auslandes als nationales bzw. nationalistisches Projekt angesehen und im Wahlkampf 1972 klangen entsprechende Töne auch an („Deutsche, wir können stolz sein auf unser Land“); vgl. Peter Hoeres, Außenpolitik und Öffentlichkeit. Massenmedien, Meinungsforschung und Arkanpolitik in den deutsch-amerikanischen Beziehungen von Erhard bis Brandt, München 2013, S. 371-512.

${ }^{21}$ Vgl. deren Urteile bei Hans Fenske, Der Anfang vom Ende des alten Europa. Die alliierte Verweigerung von Friedensgesprächen 1914-1919, München 2013.

${ }^{22}$ Vgl. an neueren historiografischen Einordnungen des Versailler Vertrages Manfred F. Boemke/Gerald D. Feldman/Elisabeth Gläser (Hrsg.), The Treaty of Versailles. A Reassessment After 75 Years, Washington 1998; Hans-Christof Kraus, Versailles und die Folgen. Außenpolitik zwischen Revisionismus und Verständigung 1919-1933, Berlin 2013; Gerd Krumeich (Hrsg.), Versailles 1919. Ziele - Wirkung - Wahrnehmung, Essen 2001.

${ }^{23}$ Vgl. Lothar Gruchmann, Nationalsozialistische Großraumordnung. Die Konstruktion einer „deutschen Monroe-Doktrin“, Stuttgart 1962.

${ }^{24}$ Vgl. Hermann Rauschning, Die Revolution des Nihilismus. Kulisse und Wirklichkeit im Dritten Reich, Zürich/New York 1938. 
Dritten Reiches, war von deutscher Seite ein Kampf gegen den atlantisch dominierten liberalen Westen“ (S. 323). Vom antibolschewistischen Kreuzzug, vom Ziel des „Lebensraums im Osten“ finden sich nur Spurenelemente, weil es anders zum Konzept nicht gepasst hätte. Der von Doering-Manteuffel bereits einmal erprobte Ansatz des Weltbürgerkriegs, mit Deutschland als Zentrum, hätte hier komplexitätssteigernd wirken können ${ }^{25}$. Denn der Gegensatz liberal versus antiliberal vereinfacht den ersten Abschnitt des Weltbürgerkriegs doch zu sehr, an dem mit Liberalismus, Bolschewismus und Faschismus mindestens drei wirkmächtige Ideologien als gesellschaftliche Ordnungskräfte beteiligt waren.

Dass das geistige Klima in der Bundesrepublik in den 1960er Jahren liberal geworden sein soll (S.341), ist mit Blick auf die Hochschulen, in denen das Klima nun eher illiberal wurde, und mit Blick auf die Vielgestaltigkeit und Heftigkeit der Debatten, der Pluralität der Zeitschriftendiskurse ${ }^{26}$ und nicht zuletzt der von Doering-Manteuffel selbst angeführten Kritiken von rechts und links am Zustand der Bundesrepublik doppelt abwegig. Die Restauration der Restaurationsthese entspringt jedenfalls wiederum eher einer zeitgenössischen Kritik, derjenigen von Walter Dirks ${ }^{27}$, die Doering-Manteuffel anführt, als einer der geschichtswissenschaftlichen Detailforschung Rechnung tragenden Perspektive.

Zeitgeschichtsschreibung sollte heutzutage nicht mehr vorrangig zur Affirmation einer westlichen Einbindung der Deutschen dienen. Wie die Sonderwegstheorie verstellt dies den nüchternen Blick auf disparate Phänomene und ungewöhnliche Verwandtschaften. Der Aufstieg der Eugenik etwa war eben gerade nicht an Nationen oder Systeme gebunden. Damit soll die Differenz zwischen Rechtsstaaten und Diktaturen (und etwa die höchst unterschiedlichen Konsequenzen der Eugenik in unterschiedlichen Systemen) keineswegs in Abrede gestellt werden, sie erscheint für problembewusste, innovative Analysen aber einigermaßen banal. Die analytisch-normative Doppelorientierung führt zu einer whigistischen Triumphgeschichte des Westens ${ }^{28}$, die nicht nur blind gegenüber der Schadensbilanz der westlichen Modernisierung ist, sondern auch ein allzu grobes Raster über die Komplexe (deutscher) Geschichte legt. Die einseitige Westorientierung der Geschichtswissenschaft fällt in dem Bestreben, Geschichte als Legitimationsquelle von Werturteilen zu funktionalisieren, hinter Webers Objektivitätsaufsatz zurück ${ }^{29}$.

${ }^{25}$ Vgl. Thomas Nipperdey/Anselm Doering-Manteuffel/Hans-Ulrich Thamer (Hrsg.), Weltbürgerkrieg der Ideologien. Antworten an Ernst Nolte. Festschrift zum 70. Geburtstag, Berlin 1993, und darin Doering-Manteuffels Aufsatz: Ernst Noltes „Deutschland und der Kalte Krieg“ im zeitgenössischen Kontext der frühen siebziger Jahre, S. 315-329.

${ }^{26}$ Vgl. Friedrich Kießling, Die undeutschen Deutschen. Eine ideengeschichtliche Archäologie der alten Bundesrepublik 1945-1972, Paderborn 2012.

${ }^{27}$ Vgl. Walter Dirks, Der restaurative Charakter der Epoche, in: Frankfurter Hefte 5 (1950), S. 942-954.

28 „Dreimal - 1918, 1945, 1989/90 - triumphierte das Ordnungsmodell des liberalen Westens über einen Gegner, der den Anspruch erhoben hatte, es niederzuringen“ (S. 324).

${ }^{29}$ Vgl. Max Weber, Die „Objektivität" sozialwissenschaftlicher und sozialpolitischer Erkenntnis, in: Archiv für Sozialwissenschaft und Sozialpolitik 19 (1904), S. 22-87. 
Begleitet wird dies von einer neuen Theoriearmut; theoretische Anschlüsse sind jenseits einiger Anklänge an die Totalitarismus- und Modernisierungstheorie nicht erkennbar. Warum wird nicht auch für geschichtswissenschaftliche Synthesen einmal die Systemtheorie Niklas Luhmanns - und sei es in undogmatischer Teiladaption - erprobt? Mit ihrer Hilfe könnte man bei aller berechtigten Kritik am soziologischen Konstrukt einer Weltgesellschaft ${ }^{30}$ im Hinblick auf die Ausdifferenzierung der Funktionssysteme - und die Entdifferenzierungsprozesse im Gefolge des Nationalismus und in den Diktaturen ${ }^{31}$ - ganz andere Zusammenhänge globalgeschichtlich herstellen und analytisch überzeugendere Kategorien zum Einsatz bringen als die sehr groben Dichotomien liberal-antiliberal, westlichnicht-westlich (immer im Sinne von Gut und Böse), die letztlich nichts anderes als eine Kombination von Schwundstufen der Modernisierungs- und der Totalitarismustheorie darstellen. Der borussischen Geschichtsschreibung wurde mit guten Gründen die einseitige Orientierung am kleindeutschen Nationalstaat vorgeworfen. Man sollte den strukturell analogen Fehler in Gestalt der analytischen Westernisierungs-Teleologie nicht wiederholen. Damit ist gerade nicht eine politische Aussage über die Rolle der Bundesrepublik Deutschland in den - im Übrigen ständig ostwärts erweiterten - transatlantischen Institutionen und Wertegemeinschaften getroffen. Für diese Westbindung und erst recht für Rechtsstaatlichkeit, Demokratie und Pluralismus gibt es gute Gründe, die aber nicht einer Funktionalisierung der Geschichte zu einer homogenisierenden teleologischen Großerzählung bedürfen, zumal eine solche politisch-pädagogische Funktionalisierung sich in ihrer Relevanz selbst aufhebt, wie Thomas Nipperdey in einem scharfsinnigen Aufsatz Anfang der 1970er Jahre gezeigt hat ${ }^{32}$.

\section{III.}

Das Konzept der Zeitbögen als Abkehr von der Fixierung auf politische und kalendarische Zäsuren ist im Grundsatz durchaus zu begrüßen, zumal auch die suggestive Zäsur der Jahrtausend- beziehungsweise Jahrhundertwende relativiert wird. Weder „9/11“ noch andere Ereignisse im Umfeld des Milleniums haben sich bisher als zentrale politik- und gesellschaftsgeschichtliche Zäsuren der deutschen

\footnotetext{
${ }^{30}$ Vgl. dazu Niklas Luhmann, Die Gesellschaft der Gesellschaft, Frankfurt a. M. 1998, Bd. 1, S. $145-171$.

${ }^{31}$ Vgl. Sigrid Meuschel, Legitimation und Parteiherrschaft. Zum Paradox von Stabilität und Revolution in der DDR 1945-1989, Frankfurt a. M. 1992.

${ }^{32}$ Vgl. Thomas Nipperdey, Über Relevanz, in: Ders., Gesellschaft, Kultur, Theorie. Gesammelte Aufsätze zur neueren Geschichte, Göttingen 1976, S. 12-32. Auf welche volkspädagogischen Abwege die Westernisierungs-Teleologie führen kann, zeigt die beharrliche Weigerung Heinrich August Winklers, sich inhaltlich ernsthaft auf den multiperspektivischen Ansatz Christopher Clarks zur Erklärung des Ausbruchs des Ersten Weltkrieges einzulassen. Vgl. Heinrich August Winkler, Die Kontinuität der Kriegspartei, in: Frankfurter Allgemeine Zeitung vom 25. 8. 2014, S. 15; ders., Die Hundert-Männer-Geschichte, in: Süddeutsche Zeitung vom 29.8. 2014, sowie die Kritik von Dominik Geppert, Die Geschichte ist schon weiter, in: Süddeutsche Zeitung vom 25.8.2014, S. 12.
} 
Geschichte herauskristallisiert ${ }^{33}$. Die inhaltliche Bestimmung der Zeitbögen, bei der die deutsche Teilung samt Vertreibung und Integration ebenso wie die Zerstörung der Städte und ihr neu gestaltender Wiederaufbau eigentümlich abwesend sind, sollte sich aber von einer anachronistischen und erkenntnishemmend wirkenden analytisch-normativen Verengung auf die Westernisierung lösen und für weitere Perspektiven öffnen: für die Ausdifferenzierung der gesellschaftlichen Funktionssysteme, für die dialektischen Prozesse von Globalisierung und Deglobalisierung ${ }^{34}$ beziehungsweise Re-Nationalisierung, für die Gouvernementalität der Postmoderne mit neuen Regimen der Normierung und Verhaltenssteuerung (beispielsweise Gender Mainstreaming, Diversity, Inklusion) ${ }^{35}$ und nicht zuletzt für die Medialisierungsschübe, die man als spezifische Zeitbögen je eines Politik und Gesellschaft prägenden und alltagsstrukturierenden Leitmediums (Radio, Fernsehen, Internet) fassen könnte ${ }^{36}$. Mit dieser offeneren, zwischen Zeitbögen und Trends changierenden Konzeption könnte dann tatsächlich ein frischer Blick auf die deutsche Geschichte im 20. Jahrhundert und auf unsere Gegenwart entstehen. Sieht man Zeitgeschichte nicht nur wie Hans Rothfels als „Epoche der Mitlebenden ${ }^{\text {‘37 }}$, sondern als Epoche der von der untersuchten Geschichte noch unmittelbar Betroffenen, wäre dies keine schlechte Option.

${ }^{33}$ Vgl. dazu Herbert, Geschichte Deutschlands im 20. Jahrhundert, S. 1238-1252.

${ }^{34}$ Vgl. Harold James, Continuities and Structural Breaks in German Economic History in the Twentieth Century, in: Anselm Doering-Manteuffel (Hrsg.), Strukturmerkmale der deutschen Geschichte des 20. Jahrhunderts, München 2006, S. 121-131.

${ }^{35}$ Vgl. Andreas Rödder, Wohin führt die Kultur der Inklusion, in: Frankfurter Allgemeine Zeitung vom 4. 7.2014, S. 6 .

${ }^{36}$ Vgl. zur Analyse von Medialisierung Frank Bösch/Norbert Frei (Hrsg.), Medialisierung und Demokratie im 20. Jahrhundert, Göttingen 2006; Frank Bösch/Peter Hoeres (Hrsg.), Außenpolitik im Medienzeitalter. Vom späten 19. Jahrhundert bis zur Gegenwart, Göttingen 2013; Klaus Arnold u.a. (Hrsg.), Von der Politisierung der Medien zur Medialisierung des Politischen? Zum Verhältnis von Medien, Öffentlichkeit und Politik im 20. Jahrhundert, Leipzig 2010.

${ }^{37}$ Hans Rothfels, Zeitgeschichte als Aufgabe, in: VfZ 1 (1953). S. 1-8, Zitat S. 2. 